\title{
A behavioral field analysis of adjunctive activities
}

\author{
NICHOLAS R. WHITE and PAUL T. P. WONG \\ Trent University, Peterborough, Ontario K9J 7B8, Canada
}

\begin{abstract}
Food-deprived rats were tested under a series of fixed-time and extinction schedules to determine whether the food-cup response and adjunctive drinking would be affected by the degree of restriction in the behavioral field. The results show that the food-cup response was higher during all schedules when rats had restricted access to various adjunctive activities and that biting and sand digging occurred more frequently than drinking.
\end{abstract}

When rats or other animals are trained under certain interval or ratio schedules for food reinforcement, they tend to engage in behaviors that are seemingly unrelated to reinforcement contingency. These behaviors include wood chewing (Roper, 1978), air licking (Meldenson \& Chillag, 1970), and wheel running (Levitsky \& Collier, 1968). The most extensively studied of these adjunctive behaviors is drinking. Adjunctive drinking has been termed "schedule-induced polydipsia," because the amounts consumed are much greater than amounts the animal would usually drink (Falk, 1961, 1969; Reynolds, Kenny, \& Wright, 1977). Schedule-induced drinking has been shown to be dependent on food deprivation (Roper \& Nieto, 1979) and on schedules in which the rate of food presentation is neither very short nor very long (Falk, 1966; Flory, 1971; W. Freed \& Meldenson, 1979). Adjunctive behavior tends to occur early in the interval (Staddon \& Ayers, 1975; Staddon \& Simmelhag, 1971).

One adjunctive behavior may be substituted for another, if opportunities for alternative behavior are available. Laites, Weiss, and Weiss (1969) found that rats nibbled their tails if denied access to a wood block, once adjunctive wood chewing had been established. Also E. Freed and Hymowitz (1969) found that rats substituted paper shredding for drinking, even though the water tube was still accessible and adjunctive drinking reappeared when the paper was removed. Others, however, found that wheel running and drinking were not ready substitutes for each other (i.e., Levitsky \& Collier, 1969). Roper (1978) reported that if rats had access to both a running wheel and a drinking tube, more drinking occurred under an FI 30-sec schedule, but wheel running predominated under an FI 60-sec schedule.

The present study adopted the behavioral field approach (Wong, 1977, 1978a, 1978b) to studying

Nicholas R. White is now at the University of Toronto. This study was supported by a research grant from the Natural Sciences and Engineering Research Council of Canada to the second author. adjunctive activities. The main purpose was to determine whether the food-cup response and adjunctive drinking would be affected by the degree of restriction of the behavioral field. It was predicted that both the food-cup response and adjunctive drinking would be higher in a restricted than in an unrestricted behavioral field. We were also interested in whether different adjunctive activities would predominate under different schedules.

\section{METHOD}

\section{Subjects}

Ten male hooded Walker-Walker rats served as subjects. All had been bred and reared in the Trent University animal laboratory and were experimentally naive. They were between 80 and 120 days old at the start of the experiment and had been reduced to $80 \%$ of their free-feeding body weights.

\section{Apparatus}

All testing took place in a $20 \times 20 \times 22 \mathrm{~cm}$ modified Skinner box constructed of sheet metal and Plexiglas. The manipulanda consisted of (1) a $5.1 \times 2.5 \mathrm{~cm}$ bar mounted on the wall, (2) a recessed $4.5 \times 4.5 \times 4 \mathrm{~cm}$ food cup, (3) a $5.7 \times 7.7 \times 5.0 \mathrm{~cm}$ recessed sandbox, (4) a water bottle, (5) a $9.8-\mathrm{cm}$ chain pull with a $1.7-\mathrm{cm}$ ring on the end, suspended from the ceiling, and (6) an $8.0 \times 5.7 \mathrm{~cm}$ fur model seal (available in Canadian gift shops), attached to the floor in an upright position. Each time a rat entered the food cup, a timer was activated. Any contact with the sand in the sandbox was recorded through a modified drinkometer circuit (Wong, Roach, \& Osborne, 1975). Contact with the water bottle was recorded by a drinkometer.

A Gerbrands food dispenser delivered .045-g Noyes pellets into the food cup on a schedule determined by a tape recorder. The entire test apparatus was enclosed in an insulated compartment and illuminated by a $7.5-\mathrm{W}$ lamp. An exhaust fan provided ventilation and ex traneous noise.

\section{Design and Procedure}

Half of the subjects had access to all manipulanda; the others did not have access to the model, sandbox, and chain after baseline testing. All animals received three sessions of baseline testing, in which no food reinforcement was given, and five daily sessions each of fixed-time (FT) $2 \mathrm{~min}$, FT $3 \mathrm{~min}$, FT $4 \mathrm{~min}$, and extinction. During FT testing, a food pellet was automatically delivered to the food cup, regardless of the subject's behavior. Each session lasted $20 \mathrm{~min}$. The experimenter observed and recorded the amount of time spent in grooming and model biting. Time spent in other behaviors was automatically recorded. 


\section{RESULTS AND DISCUSSION}

Figure 1 depicts the mean time spent by all subjects in each behavioral category averaged over all sessions. There were no reliable group differences or schedule effects in adjunctive drinking. In fact, adjunctive drinking was almost as infrequent as adjunctive barpressing. The low level of adjunctive drinking may be due to the insufficient number of testing sessions. Food-cup entry time was higher under the restricted-access condition $[F(1,8)=27.02, p<.001]$. Food-cup entry time for the last two sessions of baseline and each schedule and the first two and the last two sessions of extinction are shown in the lower panel of Figure 2. It is clear that, although the two groups did not differ during baseline, rats that had more access to adjunctive manipulanda spent less time entering the food cup during both FT schedules and extinction. If one considers food-cup entry as an operant because the appropriation of food reinforcement is contingent on food-cup entry, then the present study further demonstrates that operant behavior is affected by opportunities for adjunctive activities (Cook \& Singer, 1976; Keehn \& Riusech, 1979).

Figure 1 also shows that sand digging and model biting were much more dominant than drinking. Time spent in sand digging and model biting in different schedules is shown in the upper panel of Figure 2. All time scores were subjected to a logarithmic transformation in statistical analyses.

Model biting increased with interval length and peaked during extinction, as supported by a significant schedule effect $[F(5,20)=12.29, p<.001]$. Therefore, model biting differs from adjunctive drinking, which typically disappears during extinction (Falk, 1971). Model biting may be considered a form of scheduleinduced aggression that occurs under schedules that involve long periods of frustrative nonreward (i.e., Azrin, Hutchinson, \& Hake, 1966; Thompson \& Bloom, 1966; Wong, 1977).

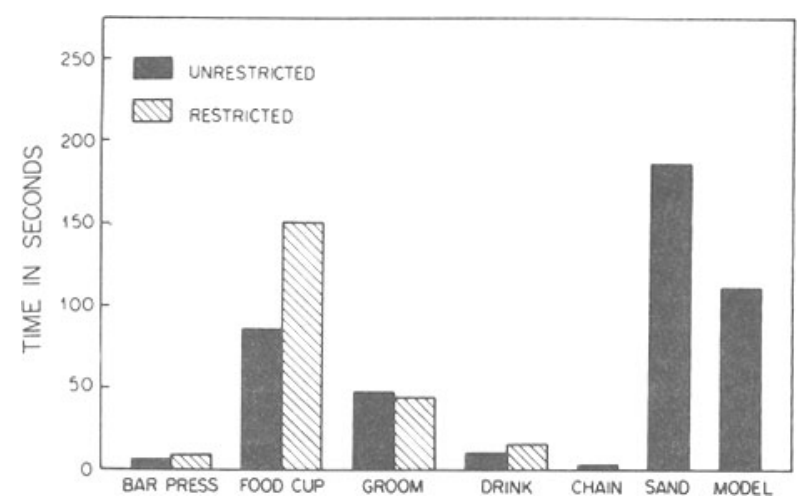

Figure 1. Mean time spent in each type of behavior. (Note that chain, sand, and model were available in the unrestricted condition only.)

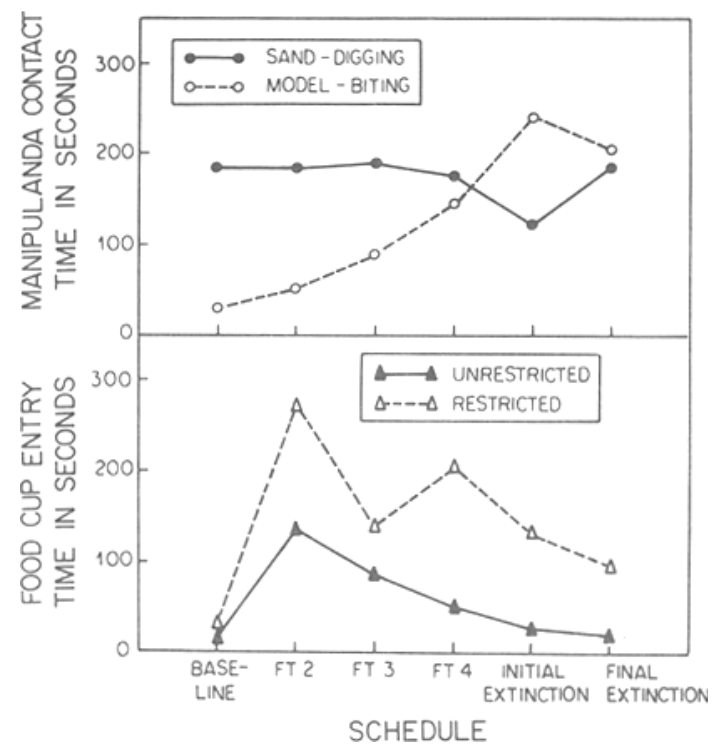

Figure 2. Top Panel: Mean time spent sand digging and model biting in the unrestricted condition under different schedules. Bottom Panel: Mean time spent in the food cup in both restricted and unrestricted conditions under different schedules.

Sand digging was less sensitive to schedule changes. Although there was a reliable schedule effect $[F(5,20)=$ $3.73, \mathrm{p}<.05]$, it seems to be largely due to a dip in digging during the initial two sessions of extinction. The relative insensitivity of sand digging to schedule changes in the present study is at variance with previous findings that extinction of a food-rewarded response induced sand digging (Wong, 1978a, 1978b). However, in the previous studies, schedule-induced sand digging was observed only after consistent reinforcement of an instrumental response.

The main findings of the present study are that the food-cup response during both FT schedules and extinction was affected by the degree of restriction of opportunities for various adjunctive activities and that model biting and sand digging had higher probabilities of occurring than adjunctive drinking regardless of the schedules.

\section{REFERENCES}

Azrin, N. H., Hutchinson, R. R., \& Hake, D. F. Extinction induced aggression. Journal of the Experimental Analysis of Behavior, 1966, 9, 191-204.

Cook, P., \& Singer, G. Effects of stimulus displacement on adjunctive behavior. Physiology \& Behavior, 1976, 16, 79-82.

FALK, J. L. Production of polydipsia in normal rats by an intermittent food schedule. Science, 1961, 133, 195-196.

FALK, J. L. Schedule induced polydipsia as a function of fixed interval length. Journal of the Experimental Analysis of Behavior, 1966, 9, 37-39.

FALK, J. L. Conditions producing psychogenic polydipsia in animals. Annals of the New York Academy of Sciences, 1969, 157, 569-593. 
FALK, J. L. The nature and determinants of adjunctive behavior. Physiology \& Behavior, 1971, 6, 577-588.

Flory, R. K. The control of schedule induced polydipsia: Frequency and magnitude of reinforcement. Learning and Motivation, 1971, 2, 215-227.

Freed, E. X., \& HYMowitz, N. A fortuitous observation regarding "psychogenic" polydipsia. Psychological Reports, 1969, 24, 224-226.

Freed, W. J., \& Meldenson, J. Control of drinking-bout magnitude in schedule-induced polydipsia by interpellet interval duration. Animal Learning \& Behavior, 1979, 7, 489-492.

Keenn, J. D., \& RiUsech, R. Schedule induced drinking facilitates schedule-controlled feeding. Animal Learning \& Behavior, $1979,7,41-44$.

LAites, V. G., WEiss, B., \& Weiss, A. B. Further observations on overt "mediating" behaviors and the discrimination of time. Journal of the Experimental Analysis of Behavior, 1969, 12, 43-57.

Levitsky, D., \& Collier, G. Schedule induced wheel running. Physiology \& Behavior, 1968, 3, 571-573.

Meldenson, J., \& Chillag, D. Schedule-induced air licking in rats. Physiology \& Behavior, 1970, 5, 535-537.

REYNolds, T. J., KenNY, J. T., \& Wright, J. W. A developmental study of schedule-induced polydipsia in the rat. Learning and Motivation, 1977, 8, 284-288.

ROPER, T. J. Diversity and substitutability of adjunctive activi- ties under fixed-interval schedules of food reinforcement. Journal of the Experimental Analysis of Behavior, 1978, 30, 83-96.
ROPER, T. J., \& Nieto, J. Schedule-induced drinking and other behavior in the rat as a function of body-weight deficit. Physiology \& Behavior, 1979, 23, 673-678.

Staddon, J. E. R., \& AYers, S. L. Sequential and temporal properties of behavior induced by a schedule of periodic food delivery. Behaviour, 1975, 54, 26-49.

Staddon, J. E. R., \& Simmelhag, V. L. The "superstition" experiment: A reexamination of the implications for the principles of adaptive behavior. Psychological Review, 1971, 78, 3-43.

Thompson, T., \& Bloom, W. Aggressive behavior and extinction induced response rate increase. Psychonomic Science, 1966, 5, 335-336.

Wong, P. T. P. A behavioral field approach to instrumental learning in the rat: I. Partial reinforcement effects and sex differences. Animal Learning \& Behavior, 1977, 5, 5-13.

Wong, P. T. P. A behavioral field approach to instrumental learning in the rat: II. Training parameters and a stage model of extinction. Animal Learning \& Behavior, 1978, 6, 82-93. (a)

Wong, P. T. P. A behavioral field approach to operant conditioning: Extinction-induced sanddigging. Bulletin of the Psychonomic Society, 1978, 12, 203-206. (b)

Wong, P. T. P., Roach, T., \& Osborne, B. A sand digging apparatus for rats. Behavior Research Methods \& Instrumentation, 1975, 7, 34-36.

(Received for publication September 23, 1982.) 